\title{
Role of Liners in Restorative Dentistry
}

\author{
Madhulika Shayamsundarji Chandak ${ }^{1}$, Manoj Chandak², Pradnya Prashant Nikhade ${ }^{3}$, \\ Chanchal Kishor Rathi ${ }^{4}$, Samrudhi Sunil Khatod ${ }^{5}$, Saurabh Dinesh Rathi ${ }^{6}$
}

\begin{abstract}
1Department of Conservative Dentistry, Sharad Pawar Dental College, Sawangi, Wardha, Maharashtra, India. 2Department of Conservative Dentistry and Endodontics, Sharad Pawar Dental College, Sawangi, Wardha, Maharashtra, India. ${ }^{3}$ Department of Conservative Dentistry and Endodontics, Sharad Pawar Dental College, Sawangi, Wardha, Maharashtra, India. ${ }^{4}$ Department of Conservative Dentistry, Sharad Pawar Dental College, Sawangi, Wardha, Maharashtra, India. ${ }^{5}$ Department of Conservative Dentistry and Endodontics, Sharad Pawar Dental College, Sawangi, Wardha, Maharashtra, India. ${ }^{6}$ Department of Conservative Dentistry and Endodontics, Sharad Pawar Dental College, Sawangi, Wardha, Maharashtra, India.
\end{abstract}

\section{ABSTRACT}

\section{BACKGROUND}

Tooth has two main organs- pulp and dentin which remain closely integrated. They depend on each other both functionally and anatomically, throughout the life of tooth. Protection of these tissues is important from trauma during cavity preparation or tooth preparation, from caries, from mechanical forces, from chemicals produced by bacteria and from galvanic shock and thermal injury. The main aim of the operative dentistry is to protect this tissue and preserve tooth vitality.(1) The purpose of a restoration is to replace missing tooth structure and provide the pulp adequate strength and protection from external insults. Sometimes, the restorations and $\backslash$ or the restorative material is incapable of providing this property; hence, an auxiliary material such as liner and base or sub-base is required, which can fulfil this function. The material used to protect this tissue is called pulp protecting material or pulp capping material. For many decades clinicians have been using liners and bases under restorations. The type of auxiliary material to be used depends upon the minimum dentinal thickness which is left between the surface of the cavity and pulp. This is known as remaining dentin thickness (RDT). Dentin has excellent buffering capacity to neutralize the effects of cariogenic acids, and insulates the pulp from temperature increases during cavity percolation. The single most important criterion for protecting pulp is remaining dentinal thickness which is dependent upon the depth of cavity preparation.(2)

\section{KEY WORDS}

Liner, Base, Calcium Hydroxide
Corresponding Author: Madhulika Shayamsundarji Chandak, Datta Meghe Institute of Medical Sciences, Radhikabai F-6, Sawangi-442001, Maharashtra, India. E-mail: chandakmadhulika40@gmail.com

DOI: $10.14260 /$ jemds/2020/409

Financial or Other Competing Interests: None.

How to Cite This Article:

Chandak MS, Chandak M, Nikhade PP, et al. Role of liners in restorative dentistry. J. Evolution Med. Dent. Sci. 2020;9(25):18811886, DOI: 10.14260/jemds/2020/409

Submission 05-01-2020,

Peer Review 22-05-2020,

Acceptance 29-05-2020,

Published 22-06-2020. 


\section{BACKGROUND}

\section{Terminologies and Classification}

The term liner is relatively a thin layer of material which is used to protect the pulp and dentin. It provides a barrier against remaining reactants diffused from restoration and / or oral fluids and may enter leaky tooth restoration interfaces.(3) According to Marzouk et al cavity liners is defined as filmforming materials that carry beneficial agents, which generate their larger film thickness (up to $25 \mu \mathrm{m}$ ) and frequently applied to dentine only. According to Ferracane a liner is a material which is applied in a thin layer used for sealing the dentinal surface and walls of the cavity, contrary to the invasion of microbial overload or nuisances from restorative procedures. The main function is to provide electrical insulation and thermal protection to the pulp. The thickness of cavity liner is $0.5 \mathrm{~mm}$. And it is applied in a thin layer on the prepared cavity surface.(4) It provides protection for the tooth from the dental filling material, intra-oral fluids, and ultimately from the outside environment. But normally it does not offer adequate thickness, rigidity, or strength to be used alone in a deep cavity, (Weiner).(2)

The greatest need for liner is in case with metallic restorations, Which preparations is extended pulpally, and not properly attached to tooth structure and non-insulating, example is amalgam, cast gold and with other indirect restorations. It can be classified as thin film liners which further divided into solution liners and suspension liners. And thicker liners which is basically used for pulpal medication and thermal protection and is also known as cement liners. ${ }^{(3)}$

Base (cement bases 1 to $2 \mathrm{~mm}$ ) is the material which is applied over the axial and pulpal wall. And it acts as a substitute for lost dentin. It provides thermal protection for dental pulp and also improved mechanical support for the restoration. It helps in allocating local stresses across from the restoration to the underlying dentin surface. It also helps to provide resistance against breakage of thin dentin over the pulp during amalgam condensation procedures, And during cementation procedures of indirect restorations. Cast metal restorations may also get advantages of proper fit on sound dentin peripheral to the lined and/or based regions. These seats may help in distributing stresses laterally to sound dentin and away from weaker underlying structure.(3)

\section{Objectives of Pulpal Protection}

It has been said that material use for the restorative purpose should be use cautiously. Because sometimes the material used for this purpose are danger to the biological tissue and causes sensitivity problems after treatment. To avoid such problems protective layer in the form of auxiliary material is applied on the tooth surface before the permanent restoration is placed. The buffering action of this protective layer helps to decrease or even abolish postoperative sensitivity. It has been considered that micro-organisms present in the mouth and their by-products enter through the margin at the toothrestoration interface by the capillary action of oral fluids, And cause the problem referred to as micro leakage.(3) Microleakage can be defined as the clinically unnoticeable passage of micro-organisms and their by-products, fluids, molecules or ions from the oral environment along the various cracks present in the cavity restoration interface. It is also occurred due to insults which cause inwards and outward movement of fluid flow, within dentinal tubules.(5) The pressure change is sensed by mechanoreceptors within the pulp and the patient experiences sensitivity. Pulp-dentin complex can be inflamed due to the microleakage of chemical nuisances from the dental materials or microbes occurs. sealing of outer ends of dentinal tubules along with cavity preparation wall is utmost important to protect against this irritants.(6) The influx of bacteria can cause difficulties including postoperative sensitivity, marginal staining, secondary caries, pulpal inflammation, pulpal necrosis, periodontal disease and possibly the subsequent need for endodontic therapy.(7) According to Camps et al it has been determined that bacteria existing inside the tooth structure is responsible for pulpal reaction.(8) After understanding the concept of 'buffer' layer, Yoshima et al. presented that closing the dentinal tubules totally desensitize the dentin, and eradicate postoperative sensitivity. (9) Other reasons for the post-operative sensitivity is contact of unlike metals, dehydration and thermal conductivity occurs cavity preparation.(9) Therefore, to prevent the placement of liners/bases in response to reduce the microleakage and their consequences gives the beneficial effects.(10) while using this material in patients it should be biocompatible is important. 'Biocompatibility' has been defined as a material's ability to elicit an appropriate biological response when in contact with the body. Possible reactions can be classified as toxic, inflammatory, allergic or mutagenic. The pulp has the ability to lay down more dentine ('reparative' or 'tertiary' dentine) when irritated by caries, cavity preparation or as a result of the interaction between the tooth and the restorative material. There are materials that promote reparative dentine formation and others that can cause pulpal damage.

Remaining dentin thickness approximately $2 \mathrm{~mm}$ of dentin or an equivalent thickness of materials should exist to protect the pulp. This thickness is not always possible, but $1-1.5 \mathrm{~mm}$ of insulation is accepted as a practical thickness. As the tooth preparation extends closer to the pulp, a thick liner or a base is used to augment dentin to the proper thickness range. The remaining dentin thickness estimated to be necessary for protection of the dental pulp against injury or inflammation has changed over the years.(11)

\section{Clinical Significance}

If there is shallow cavity and remaining dentin thickness is $>2$ $\mathrm{mm}$ no effect is seen in number of odontoblast reduction and there is minimal secretion of reactionary dentin. The treatment options for this is solution liner only-to be given on all internal surfaces of the cavity under metallic restoration, no liner $\backslash$ base for tooth coloured restoration (such as GIC, composite resins) ${ }^{(12)}$ If there is deep cavity and remaining dentin thickness is $0.5 \mathrm{~mm}-0.25 \mathrm{~mm}$ slight reduction in number of odontoblasts is seen and there is minimal secretion of reactionary dentin. In such cases, a base of thickness 0.5 $0.75 \mathrm{~mm}$ is given on the pulpal floor and axial wall only. RDT $<$ $1 \mathrm{~mm}$, the pulpal floor near to the pulp where the RDT is less than $1 \mathrm{~mm}$ is covered with suspension liner, over which base is given.(12)
} 


\section{Requirements for Liners and Bases}

1. They should be non-harmful, and it does not irritate to the pulp and other tissues.

2. It is not soluble in saliva and fluids taken into the mouth.

3. It provides good mechanical properties which fulfil the requirements filling material to be packed on liner.

4. Protect the pulp from pulpal reactions caused by different restorative material.

5. Under a large metallic restoration cement is used to provide thermal insulation to the pulp e.g. amalgam

6. Liners and bases also provides chemical protection to prevent infiltration of hazardous chemicals from the dental material to the pulp.

7. It provides electrical insulation under the metallic restoration to reduce the galvanism.

8. Optical properties for cementation of a translucent restoration (for example, a porcelain crown) the optical properties of the cement should be parallel to those of tooth substance.

9. A cement should ideally be adhesive to enamel and dentin, and to gold alloys, porcelain and acrylics, but not to dental instruments.

10. It should be bacteriostatic while inserted in a cavity with residual caries.

11. Cements should have a minimum adverse effect on the pulp.

12. For luting purposes, cements should have a low film thickness.

\section{Cavity Varnish}

It is applied in case of shallow cavities. Especially under the cavity walls of amalgam and cast metal restorations. In case of composites and glass- ionomer cements the application of cavity varnish can be avoided. Varnish coatings are formed by drying the solutions of copal or other resin dissolved in a volatile solvent. The most widely used varnish is copalite. The constituents of this is $10 \%$ copal resin and organic solvents such as ether, alcohol and acetone. Medicinal agents such as chlorobutanol, thymol, and eugenol also have been added. To produce the thin film layer the resin component kept low. Because thin film provides flexibility and dry easily as compared to thick films. This provides better sealing of the dentinal tubules and leads to good marginal adaptability, especially in the case of silver amalgam restoration till the corrosion products fill the gap. It has been seen that only 2 to 3 layer of varnish is formed over the smear layer along the tooth preparation wall, Which does not provides completely wet surfaces because smear layer contains some amount of moisture and varnish is hydrophobic in nature, And that's why single coating of varnish covers only $55 \%$ of the surface. A second layer of varnish is recommended to produce sealing up to 80 to $85 \%$.

\section{Cavity Sealers}

It is a material that provides better sealing and bonding at the restoration and cavity wall interface. The example of cavity sealers include dentin bonding agents which replaces the cavity varnish. The film thickness is $20-50 \mu \mathrm{m}$.

\section{Functions of Varnish and Sealer}

- It prevents microleakage.

- It prevents post-operative sensitivity by not allowing the ingress of fluids from the cavity margin

- It also prevents the penetration of the toxic material into the dentin from the restorative material and also prevents discoloration.

- Suspension liners: It contains calcium hydroxide, zinc oxide eugenol (Type 4)

\section{Conventional Calcium Hydroxide Liners}

$\mathrm{Ca}(\mathrm{OH})_{2}$ (Calcium hydroxide) is the material introduced in clinical dentistry in 1921 by Hermann. It is the gold standard material used for pulp protection in case of direct and indirect pulp capping treatment procedures. It is used as liners or subbase. It is available in various form such as aqueous suspensions containing organic liquid such as methyl ethyl ketone or ethyl alcohol or in aqueous solution of methyl cellulose. The methyl cellulose is used as thickening agent. It is also available in two paste form the base paste and catalyst paste (Dycal (Dentsply, Milford, DE, USA)). It is rigid selfsetting radiopaque material. The composition of base paste contains 1, 3-Butylene glycol disalicylate reacts with calcium hydroxide and zinc oxide, zinc oxide, calcium phosphate, calcium tungstate which provide radiopacity and iron oxide pigments which renders the colour.(13) The catalyst paste contains calcium hydroxide is one of the primary reactive ingredients, zinc oxide is also primary reacting material, $\mathrm{N}$ ethyl-o/p-toluene sulfonamide is the plasticizer, zinc stearate acts as accelerator. It is chemically cured fast setting material. The normal setting time is 2.5-3.5 minutes. The chemical reaction occurs between calcium hydroxide and salicylate is acid base reaction, which is responsible for the formation amorphous calcium disalicylate. The calcium hydroxide material is categorized as low strength base, so it required a suitable high strength base to increase the compressive strength before placement of final restoration(14). Calcium hydroxide liner has the antibacterial properties. Due to these properties of liners it inhibits the bacterial growth under restorations. As the bacterial overload due to caries decrease the extent of pulpal inflammation is reduced. The set material has higher solubility in aqueous medium. $\mathrm{Ca}(\mathrm{OH}) 2$ is dissociate into calcium and hydroxyl ions. Hydroxy ions are responsible to create an alkaline $\mathrm{pH}$ which creates an unfavourable environment for remaining bacteria. Hydroxyl ions are highly oxidant free radicals that show high reactivity, And these proves the antimicrobial properties of material. These characteristics of material helps in case of deep cavities where the chances of carious exposure of the pulp or mechanical exposure due to traumatic injuries may occur. As It is biocompatible material. The mechanism action of calcium hydroxide for the formation of mineralized barrier, is the product of irritation of pulp tissue by the cement, possibly mediated by activation of transforming growth factor beta and cellular growth factor. Though it has many potential effects there has been some significant drawbacks. It has low compressive strength, low modulus of elasticity, so it is not to be used in critical areas to support the restoration. It has low thermal conductivity. It has high solubility in water so it can dissolve partially or totally under the poor sealed tooth restoration interface.(13) 


\section{Light Cured Calcium Hydroxide}

It is available as single constituent liner. It contains $\mathrm{Ca}(\mathrm{OH})_{2}$ which is hardened by visible light cure through polymerization reaction. It is mainly introduced to overcome the drawbacks of the traditional chemical cure calcium hydroxide. It is set on command with the light cure unit. It provides improved strength, decreased solubility in acids and less solubility in water. The composition of light cured calcium hydroxide liner consists of calcium hydroxide and barium sulphate dispersed in a urethane dimethacrylate resin containing initiators and accelerators activated by visible light. It contains polymeric resin allows better bonding with composite resin cement.

\section{Zinc Oxide Eugenol}

The ADA specification no of zinc oxide eugenol cement is 30 . This cement used extensively date back since 1890. It was introduced due to its easy handling characteristic and manipulative even in the presence of moisture and well tolerated by the pulp tissue. Composition: The powder mainly constitutes of zinc-oxide (70\%) and rosin $(29 \%)$ to decrease the brittleness of set material, zinc acetate $0.7 \%$ as accelerator and zinc stearate $1 \%$ as plasticizer. The liquid mainly contains eugenol which is organic liquid, is chemical essence of oil of cloves. It is weak acid which has bactericidal activity and obtundent effect. The necessities for ZOE as a base are given in ISO 3107-2004, under the category of Type 3. It is least noxious to all dental materials. It has sedative effect on the pulp at ph 7.(14) If eugenol is present in higher concentration it causes toxic reaction to the pulp. The setting of ZOE cements is a chelation reaction in which an amorphous, zinc eugenolate is formed. The setting reaction is accelerated by increases in temperature or humidity. The moisture present in dentin causes released more eugenol to form concentration gradient that inhibits the bacteria but does not gives adverse effect to the pulp. Lower powder liquid ratio provides an excellent marginal seal, even though $\mathrm{ZOE}$ is not bond to the tooth. The advantage of sealing is prevention of diet-derived substrate from reaching the micro-organisms found below the restoration. This results in the reduction of both acid production and of the formation of secondary caries. Essentially, ZOE inhibits bacterial cell metabolism, the end result being a low incidence of postoperative sensitivity. It is used as base material having compressive strength of 5.5 to 39 $\mathrm{MPa}$ and strength reaches maximum in about 12 to 15 minutes. The zinc oxide eugenol has excellent thermal properties and is approximately the same as human dentin. Zinc oxide eugenol is marketed as cavity liner but not as base. The zinc oxide eugenol base is not used under the composite restoration because eugenol inhibits the polymerization reaction.(16)

\section{Zinc Phosphate}

Zinc phosphate cement is the oldest luting cement introduced way back in 1800s by Dr. Otto Hoffman. The ADA specification no is 96. It has excellent clinical performance, in spite of many inherent drawback such as chemical irritation to the pulp, lack of adhesion, lack of antibacterial action and high solubility in oral fluids. It has two component powder and liquid.

\section{Composition}

Powder contains zinc oxide approximately $90.2 \%$ and magnesium oxide $8.2 \%$ which reduce the temperature of calcination process during manufacture, other oxides $1.6 \%$ which alters the working characteristic and final properties of the cement. Bismuth trioxide imparts smoothness to the freshly mixed cement. Magnesium oxide also imparts white colour to the cement besides improving the strength. The liquid is composed aqueous solution of phosphoric acid (50 to $60 \%$ ), which is combined with aluminium and zinc $16.2 \%$ and acts buffers and partially neutralize the phosphoric acid, thus tempering the reactivity of liquid and water 33\%. The rate of acid base reaction is influenced by water. Large amount of water results in reduction of both compressive strength and tensile strength. The setting time for zinc phosphate cement is 2.5 and 8 minutes.

\section{Available Types and Their Clinical Applications}

1. Type I-Fine grained: Film thickness less than 25 -used for luting permanent metallic restorations and cementation of orthodontic bands.

2. Type II-Medium grained: Film thickness of 40 -High strength thermal insulating base.

The main advantage of zinc phosphate cement has high compressive strength initial (100-160 MPa) in first 10 minutes and reaches its final strength after 24 hours; hence it is used as base under amalgam restoration and it can withstand the condensation forces during its placement. It also has excellent thermal insulation but in thickness of $1 \mathrm{~mm}$ and poor electrical insulation due to its porous nature which accumulates moisture and encourage electron transport. Biocompatibility of cement is low. Cement has potential to cause pulpal inflammation especially in deep cavities due to the presence of acids. Hence it is mandatory to apply the cavity varnish before the placement of cement. The initial $\mathrm{pH}$ rises rapidly and reach up to 4.2 within 3 minutes after the mix and reaches at PH 6 by the end of 1 hour. And get neutralize after 48 hours. (17)

Disadvantage of zinc phosphate cement is low tensile strength (5-8 $\mathrm{MPa}$ ) and hence brittle in nature. It lacks the chemical adhesion; retention is only by mechanical means. Examples of zinc phosphate are marketed by Henry Schein Halas, Dentavision P/L and Ivoclar Vivadent P/L.(17)

\section{Glass-Ionomer Cement}

Glass ionomer cement was first introduced by Wilson and Kent in 1972. GIC is combination of the powder of silicate cement and liquid polycarboxylates and hence the name aluminosilicate polyacrylic acid (ASPA). The other name of GIC includes polyalkenoates, dentin substitute, and man-made dentin. The two main features of glass-ionomer cement which make it most acceptable restorative material in the treatment of direct pulp capping and indirect pulp capping. It bonds well to enamel and dentin and released fluoride from the glass component of the cement. Due to this it helps to reduced dentinal hypersensitivity(18). 


\section{Composition of Glass-Ionomer of Cement}

Conventional GIC is available in the form of powder and liquid. Powder is an acid soluble calcium fluoroaluminosilicate glass. It basically consists of silica, alumina, fluorides of calcium, sodium and aluminium. The function of silica is it acts as glass matrix and increases translucency. The fluoride is fundamentally acts as flux. It leached out from the set cement and provides anticariogenicity. Alumina is added in the si/Al atomic ratio of $<2: 1$ to make the glass basic enough so it becomes susceptible to acid attack and release various ions. ${ }^{19)}$

\section{Resin Modified Glass Inomer Cement}

Resin-modified glass ionomers (RMGI) were introduced to improve mechanical properties, reduce setting time, and lessen moistness sensitivity. Simply RMGIs are a hybrid of glass ionomers and composite resin. And thus comprises both acid-base and polymerizable components.

For deeper restorations that are near to the pulp but where pulp is not exposed, an RMGIC liner is a primary choice. These materials are dimensionally very stable, they bond to dentin, and release fluoride. They also decrease stress on the tooth and can inhibit microleakage. In one study there is comparative evaluation of Class I restorations lined with an RMGI liner and dentin adhesive versus dentin adhesive alone. Severity and incidence of sensitivity was found to be less in the RMGI group at both 24 hours and seven days, a critical time period for the patient's judgment of the comfort of the restoration(16). RMGI liner has also been shown to be an effective tool to decrease the micro leakage, which is one of the most frequently occurring problems for a restoration.(19)

\section{CALCIUM SILICATE BASED LINERS}

\section{Mineral-Trioxide Aggregate}

Mineral trioxide aggregate (MTA) is an exclusive material with numerous exciting clinical uses. MTA has potential and is one of the most versatile materials of this century in the field of dentistry. MTA materials have been shown to have a biocompatible nature. MTA materials have been shown to have excellent potential as pulp-capping and pulpotomy medicaments.(20)

\section{Biodentine}

It is calcium-silicate-based material which became commercially available in 2009 (Septodont) and it was specifically designed as a "dentine replacement" material. It has a wide range of applications such as endodontic repair (root perforations, apexification, resorptive lesions, and retrograde filling material in endodontic surgery) and pulp capping which can be used as a dentine substitute material in restorative dentistry. The Biodentine is available in powder and liquid form. The powder form is composed of tricalcium silicate, dicalcium silicate, calcium carbonate and oxide filler, iron oxide shade, and zirconium oxide. And the liquid consists of calcium chloride as an accelerator and a hydrosoluble polymer which serves as a water reducing agent. Fast setting time 45 minutes is the unique feature of this material. It has positive effect on vital pulp cells and stimulate the tertiary dentin formation. ${ }^{(21)}$

\section{Theracal LC (BISCO INC)}

It is introduced to overcome the drawbacks of Biodentine that is poor micromechanical bonding with the resin composite restoration. Theracal LC is Resin-Modified Calcium Silicate Pulp Protectant/Liner. It is categorized as fourth generation calcium silicate based material. It provides calcific barrier to protect the underlying pulp-dentin complex. The material sets by polymerization reaction using light activation. Due to this clinician can place and condense permanent restoration immediately in single sitting. The material also have thixotropic behaviour. The proprietary hydrophilic resin formulation creates a stable and durable liner or base. The $\mathrm{PH}$ of Theracal LC is alkaline in nature and is 10.66 at 3 hours and no significant reduction seen at 24 hours i.e. 9.85 .

\section{Indications}

It is used as pulp capping material in both direct and indirect pulp capping procedures. It may be placed directly on pulpal exposures after haemostasis is obtained-

1. Under Class I and Class II composite restorations.

2. Under other base material.

3. Under cements.

4. As an alternative to calcium hydroxide.

5. As an alternative to glass ionomer/RMGI.

6. As an alternative to cavity varnish sealer.

7. As an alternative to zinc phosphate.

8. As an alternative to IRM / ZOE (Intermediate Restorative Material).

\section{Advantages}

- It is help in the formation of reparative dentin.

- It has low solubility as compared to Biodentine, calcium hydroxide, ProRoot MTA, MTA Angelus.

- It acts as remineralizing agent

- It has better bond strength compared to Calcium hydroxide, Biodentine and comparable with GIC.

- It has anti-bacterial property.

\section{Disadvantages}

It contains polymerizable methacrylate monomers. So avoid prolonged or frequent contact with skin, oral soft tissues, and eyes. TheraCal LC is not suggested for use with patients who have a history of severe allergic reaction to methacrylate resins. ${ }^{(22)}$

\section{Endosequence BC liner}

It is a bioceramic cements that have been used for pulpal or root defect repairs in adult or paediatric teeth. It is also indicated as liner or base over the access openings in primary and permanent dentition covering any of standard dressings, spacers or obturation materials. Initial setting time is 20 seconds and initial self-curing time at 37 degree Celsius is 2.5 to 3 minutes. 


\section{RESIN-BASED FLOWABLE COMPOSITE LINER}

There has been many advantages and improvement taken place in composite resin restoration. High polymerization shrinkage is the still biggest issue. Due to increases in polymerization shrinkages it causes two main problems. That is bond failure between the restoration and tooth margin which further leads the microleakage as major consequences. For avoiding such problems and reliving stress cause due polymerization.(23) A highly flexible intermediate layer of liner between the restoration is suggested. Flowable composite is the material of choice as auxiliary liner. Due to their characteristic features like low viscosity, increased elasticity and wettability. It has better marginal adaptation with composite restoration. The first generation flowable composite resin was introduced in 1996. Recently Smart dentin replacement (SDR) (Dentsply, Konstanz, Germany) was introduced in clinical practice. The filler load present is $68 \%$ by weight. It incorporates photoactive group in modified urethane dimethacrylate resin. It has been determined that activated resin has comparatively slow radical polymerization rate. It has been noticed that photo initiators which are included affects the polymerization process. Additionally, the incorporation of activated resin results in reduction of $60-70 \%$ shrinkage stress when compared to conventional methacrylate-based resins. It is applied in bulk as single layer up to $4 \mathrm{~mm}$ thick, followed by $2 \mathrm{~mm}$ layer of composite restoration.

\section{CONCLUSIONS}

As can be seen from the above review, the material science of liners and bases is a not a finite area of study. It is an evolving situation that requires the clinician to stay abreast of the constantly changing research.

\section{REFERENCES}

[1] Christensen GJ. To base or not to base. J Am Dent Assoc 1991;122(6):61-2.

[2] Weiner R, Kugel G, Weiner L. Teaching the use of bases and liners: a survey of North American dental schools. J Am Dent Assoc 1996;127(11):1640-5.

[3] Anusavice K. Phillip's science of dental materials. $11^{\text {th }}$ edn. Philadelphia: WB Saunders 2003.

[4] Craig RG, Powers JM. Restorative dental materials. $11^{\text {th }}$ edn. St Louis, MO: Mosby 2002.

[5] Gordan VV, Vargas MA, Cobb DS, et al. Evaluation of acidic primers in microleakage of Class 5 composite resin restorations. Oper Dent 1998;23(5):244-9.

[6] Murray PE, About I, Franquin JC, et al. Restorative pulpal and repair responses. J Am Dent Assoc 2001;132(8):48291.
[7] Brannstrom M. Etiology of dentin hypersensitivity. Proc Finn Dent Soc 1992;88 Suppl 1:7-13.

[8] Camps J, Dejou J, Remusat M, et al. Factors pulpal response to cavity restorations. Dent Mater 2000;16(6):432-40.

[9] Yoshiyama M, Masada J, Uchida A, et al. Scanning electronic microscopic characterization of sensitive vs. insensitive human radicular dentin. J Dent Res 1989;68(11):1498-502.

[10] von Fraunhofer JA, Marshall KR, Holman BG. The effect of base/ liner use on restoration leakage. Gen Dent 2006;54(2):106-9.

[11] Tomer AK, Miglani A, Chauhan P, et al. Residual dentine thickness. International Journal of Applied Dental Sciences 2016;2(4):96-9.

[12] Murray PE, Lumley PJ, Franquin JC, et al. Cavity remaining dentin thickness and pulpal activity. Am J Dent 2002;15(1):41-6.

[13] Arandi NZ. Calcium hydroxide liners: a literature review. Clin Cosmet Investig Dent 2017;9:67-72.

[14] Nikahde PP, Kibe AN, Thote AP, et al. Assessment of penetration of low surface tension vehicles in dentinal tubules using stereomicroscopy. World J Dent 2019;10(2):135-9.

[15] Ferracane JL. Materials in dentistry: principles and applications. $2^{\text {nd }}$ edn. Philadelphia: Lippincott Williams and Wilkins 2001.

[16] Weston JF. Use of a resin-modified glass-ionomer (RMGI) liner in conservative direct treatment of deep caries. Compend Contin Educ Dent 2015;36(1):42-5.

[17] Paul J. Dental cements- a review to proper selection. Int J Curr Microbiol App Sci 2015;4(2):659-9.

[18] Strober B, Veitz-Keenan A, Barna JA, et al. Effectiveness of a resin-modified glass ionomer liner in reducing hypersensitivity in posterior restorations: a study from the practitioners engaged in applied research and learning network. J Am Dent Assoc 2013;144(8):886-97.

[19] Sidhu SK, Nicholson JW. A review of glass-ionomer cements for clinical dentistry. J Funct Biomater 2016;7(3):16.

[20] Mostafa NM, Moussa SA. Mineral trioxide aggregate (MTA) vs calcium hydroxide in direct pulp cappingliterature review. Online Journal of Dentistry \& Oral Health 2018;1(2):1-6.

[21] Malkondu Ö, Kazandağ MK, Kazazoğlu E. A review on biodentine, a contemporary dentine replacement and repair material. Biomed Res Int 2014;2014:1-10.

[22] Arandi NZ, Rabi T. TheraCal LC: from biochemical and bioactive properties to clinical applications. Int J Dent 2018;2018:1-6.

[23] Panchbhai A. Nano composites: past, present, and future of dentistry. In: Asiri AM, Inamuddin, Mohammad A, eds. Applications of nanocomposite materials in dentistry. $1^{\text {st }}$ edn. Woodhead Publishing 2018:181-90. 